\title{
Prophylactic Antibiotics for Third Molar Surgery: An Enigma or Panacea?
}

\author{
Kanwaldeep Singh Soodan ${ }^{1}$, Pratiksha Priyadarshni ${ }^{2}$, Nageshwar Iyer ${ }^{3}$, \\ Atul Sharma ${ }^{4}$, Vasit Khajuria ${ }^{5}$ \\ 1,2,3,4,5 (Department of Oral \& Maxillofacial surgery, M. M. Institute of Medical Sciences and Research, \\ Mullana, Ambala/ Maharishi Markandeshwar University, India)
}

\begin{abstract}
The removal of impacted third molars is a common procedure in oral and maxillofacial surgery. There is controversy about use of antibiotics as prophylaxis to prevent complications after third molar removal. Several authors claim that prophylactic antibiotics are useful in preventing postoperative complications and infections after third molar removal. Decreased pain, swelling and trismus are reported. Quality of life is stated to be higher when antibiotics are prescribed. On the other hand, additional studies were not able to demonstrate the effectiveness of antibiotic prophylaxis. The purpose of this study is to review the literature regarding the appropriateness of the routine use of antibiotics for third molar surgery.
\end{abstract}

Keywords: prophylactic, antibiotics, molar surgery

\section{Introduction}

The use of antibiotic therapy is well established in the treatment of identified infections caused by susceptible microorganisms and for prophylaxis in clean contaminated or contaminated surgery and in the prevention of sub acute bacterial endocarditis after intraoral procedures in patients after total joint implantation and in the prevention of infection in the immunocompromised patient. However although third molar surgery may usually be considered clean-contaminated and occasionally contaminated surgery, the use of routine antibiotic prophylaxis in patients undergoing such surgery is a controversial topic. It is common practice in oral and maxillofacial surgery to use antibiotics after third molar surgery. In some offices it is a universal practice to prescribe them for all patients having routine removal of impacted third molars. In considering the question "should antibiotics be used for third molar surgery?" one can give at least five possible reasons. Use antibiotics when 1) an infection is present that must be treated 2) the patient is medically compromised and requires antibiotic prophylaxis against metastatic infection 3) the patient or the patient's family demands antibiotics 4) the standard of care in the oral surgery community is to use antibiotics and hence not to use them violates this standard and when 5) the risk of postoperative infection is high and consequently prophylaxis is needed. The purpose of this article is to review the literature regarding the appropriateness of the routine use of antibiotics for third molar surgery.

\section{The Use of Prophylactic Antibiotics}

Although the use of antibiotics may be common after removal of third molars, could this practice be said to adhere to the principles of antibiotic prophylaxis? Peterson in his article has delineated these principles: 1) The surgical procedure should have a significant risk of infection. 2) Select the correct antibiotic for the surgical procedure. 3) The antibiotic level must be high. 4) Time the antibiotic administration correctly. 5) Use the shortest effective antibiotic exposure [1]. The first principle sets the criterion for antibiotic use, whereas principles 2 through 5 suggest the protocol for administration. If the routine use of antibiotics for third molar surgery is to be recommended, principle 1 must be proved. The use of antibiotic therapy without appropriate indications can result in adverse outcomes. It is clear that there is a general trend to overprescribe antibiotics as well as medications in general [2]. Some of the risks of indiscriminate antibiotic therapy include the development of resistant organisms, secondary infection, toxicity of the antibiotics, and development of allergic reactions $[3,4]$. It is estimated that $6 \%$ to $7 \%$ of patients receiving antibiotics experience some kind of adverse reaction [5]. Clearly, the advantages of antibiotic therapy in the asymptomatic patient must exceed the risk of adverse outcomes. What is the surgeon's motivation for using routine antibiotic therapy in the removal of third molars? Prevention of infection would be the most logical reason. In addition there is a large body of information in the literature concerning the reduction of morbidity other than infection, usually focusing on decreasing the incidence of alveolar osteitis and the severity of the pain, trismus, and postoperative edema. Altering the incidence of these common occurrences could be a reason for considering prophylactic antibiotic therapy. 


\section{The Presence of Infection at the Time of Surgery}

The first indication for use of antibiotics is therapeutic rather than prophylactic and will not be developed at length in this article. Treatment of an active infection includes both surgical drainage and antibiotics. Consequently, the use of antibiotics for cases in which third molars are removed in the presence of an ongoing infection is not in dispute.

\section{Antibiotic Prophylaxis for Medically Compromised Patients}

Antibiotic prophylaxis may be considered for several groups of patients including cardiovascular patients with susceptible lesions, immunocompromised patients, and patients with orthopedic prostheses. In 1990, the American Heart Association (AHA) published its most current recommendations which were based on in vitro studies as "No adequate, controlled clinical trials of antibiotic regimens for prevention of bacterial endocarditis in humans have been done" [6]. These recommendations were nonetheless accepted by most surgeons as being scientifically valid. As regards patients who are immunocompromised a article documented a 10 times greater risk of postoperative complications in human immunodeficiency virus (HIV)-positive patients undergoing dental extractions (not confined to third molars) when compared with HIV-negative patients [7]. Risk increased with the severity of the HIV infection. Antibiotic prophylaxes in this group were not disputed although there were no clear-cut guidelines. There are still no definitive recommendations from any authoritative source on the role of prophylactic antibiotics for third molar surgery in patients with orthopaedic prostheses. In 1990, the American Dental Association published a belated report of a 1987 workshop that cited insufficient data to support the effectiveness of antibiotic prophylaxis for dental procedures [8]. In 1992, the Working Party of the British Society for Antimicrobial Chemotherapy advised against routine antibiotic prophylaxis [9]. A questionnaire sent to department heads of US dental schools revealed that $54 \%$ of respondents did teach students to use antibiotic prophylaxis for these patients but 18 different regimens were recommended and individual departments within the same dental school were sometimes at variance [10]. No authoritative orthopedic organization has made a specific recommendation although individual orthopedic surgeons often request antibiotic coverage. Spread of intraoral bacteria to orthopedic prostheses has been poorly documented. A computer simulation study showed that the minuscule risk of fatal anaphylaxis in dental patients receiving prophylactic antibiotics is actually higher than the risk of late prosthetic joint infection causally related to dental therapy [11]. These investigators suggested that only dental patients undergoing procedures with a high risk of bacteraemia (periodontal therapy, impaction surgery) should receive antibiotic prophylaxis.

\section{Use of Antibiotics Because of Patient Demand}

Pressure toward early antibiotic intervention exists in our society. "Patients like quick fixes. When a doctor doesn't prescribe an antibiotic, they will look for one who will" [12]. Most patients are not aware of the risks of indiscriminate antibiotic use, such as the development of resistant strains of bacteria. In fact, people at America ingest antibiotics every day in dairy products and meat because animals are routinely given antibiotics in very large doses to prevent infection. The US Federal Drug Administration (FDA) permits milk to contain small amounts of 80 different antibiotics (they are used on dairy cows to prevent udder infections) [13]. Rarely do patients refuse antibiotics. However, the request of patients for antibiotics without medical indication is never a justifiable rationale.

\section{Risk of Postoperative Infection is High-Therefore Prophylaxis Required}

There is no consensus in the literature on whether the risk of infection with third molar surgery is high enough to warrant the use of prophylactic antibiotics. Many dental and oral surgical textbooks recommend against the use of prophylactic antibiotics for extractions, including third molar surgery unless active infection is present at the time of surgery [14-17]. Others recommend routine antibiotic use for "deep, difficult impactions for a minimum of 5 to 7 days". Of interest is the fact that none of the recommendations in these texts is referenced. Scientific journal articles offer conflicting opinions as to the efficacy of antibiotics in the prevention of postoperative infection. Most articles involve either extremely small numbers of patients in prospective studies or larger numbers in retrospective studies, none of which state whether the patients were all seen postoperatively.

\section{Literature against Antibiotic Use}

Investigators concluding that prophylactic antibiotics should not be used include Curran et al who divided 68 patients who had 133 mandibular bony impactions into two regimen groups: 1) penicillin intramuscularly 1 hour before surgery followed by oral penicillin for 4 days 2) no antibiotics [18]. No statistics were used in this report. Curran's conclusions contradicted the results because $7.8 \%$ (5 of 64) of the sockets that were treated with antibiotics got infected whereas $8.7 \%$ (6 of 69) of the sockets without antibiotics became infected. Happonen et a1 divided 136 patients who had mandibular third molar extractions into three random groups, each of which 
was given an intramuscular injection 1 hour preoperatively and 15 tablets over 5 days postoperatively [19]. Of the patients receiving penicillin, 13.6\% (6 of 44) became infected, whereas $10.6 \%$ (5 of 47) who received tinidazole and $11.1 \%$ ( 5 of 45 ) who received placebo developed infections. These differences were not statistically significant. Goldberg et a1 made the statement that " antibiotic prophylaxis is not useful in the prevention of postsurgical wound infection" however analysis of the data in this article showed that $1.1 \%$ ( 1 of 90) of patients receiving antibiotics developed infections whereas $9.4 \%$ (20 of 212) of patients who did not receive antibiotics developed infections [20]. Capuzzi et al saw no statistical difference in their 146 patients, half on postoperative amoxicillin for 4 days and half without antibiotics in terms of pain and swelling but did not comment on the infection rate [21]. This study violated a principle of antibiotic prophylaxis in that no antibiotics were in tissue before the surgery [22]. Other investigators have written editorials advising against the use of antibiotics on the basis of possible adverse reaction to the drug or on emotion [23, 24].

\section{Literature for Antibiotic Use}

Investigators who favour the use of antibiotics include those commenting on direct application within sockets as well as those favouring systemic antibiotics. Antibiotics placed directly into the socket including tetracycline, metronidazole and both lincomycin and oxytetracycline have been shown to be very effective in reducing significantly the incidence of alveolar osteitis (dry socket), whereas one prospective double blind study comparing 85 patients with neomycin/bacitracin cones with 59 controls showed a $7.1 \%$ infection rate in the antibiotic group and a 20.3\% rate in the control group [25-29]. One article other than Goldberg's is dealing with the use of systemic antibiotics in which the incidence of infection was compared in antibiotic and no antibiotic groups . Mitchell reported a 4\% (4 of 45) incidence in his tinidazole group versus a $45 \%$ (20 of 44 ) incidence in his placebo group [30]. A subsequent comparison study by the same author showed similar infection rates in groups given either of pivampicillin or tinidazole but there was no control group [31]. Four articles support the use of antibiotics on the basis of decreased trismus, swelling, pain or better wound healing but did not specifically comment on infection rates [32-35]. One randomized prospective article studied bacterial growth in third molar sockets of 120 patients: 40 with preoperative and postoperative penicillin, 40 with preoperative and postoperative scopolamine (to reduce salivary flow) and 40 with no medication. Growth of both aerobic and anaerobic bacteria within the sockets was significantly decreased in the group on penicillin [36]. Thus, a review of the literature reveals no clear-cut guidelines. Most of the articles discouraging antibiotic use are flawed in either scientific method or conclusions whereas most of those supporting antibiotic use study the problem tangentially.

\section{Conclusion}

This literature review has attempted to evaluate the use of antibiotic therapy for third molar surgery to determine the need for routine use. The incidence of postoperative infection ranges from $1 \%$ to less than $6 \%$ with most of those being minor infections. Considering that the first principle of antibiotic prophylaxis is that the surgical procedure should have a significant risk of infection, this low complication rate would not support the routine use of antibiotic prophylaxis. In addition, the potential for adverse reaction to antibiotic therapy exceeds any possible decrease in infection. Further, the available studies that have compared infection rates after use or non use of antibiotics do not show decreased infections in the antibiotic groups. Evaluation of the use of antibiotics to decrease other adverse outcomes such as alveolar osteitis, trismus, pain, and swelling tend to show little improvement with antibiotics. It is important to evaluate each study individually because as the methods of the study become less stringent, the clinical trials tend to show more benefits from antibiotics. Double blind, randomized prospective studies even in susceptible patients generally find no difference between antibiotic and control groups. In those less stringent studies finding benefits to antibiotic therapy, these benefits tend to be minimal and usually occur late in healing, when most patients are having few problems. Considering these findings, the routine use of prophylactic antibiotics in patients having removal of impacted third molars cannot be recommended.

\section{References}

[1]. Peterson LJ, Antibiotic prophylaxis against wound infections in oral and maxillofacial surgery, J Oral Maxillofac. Surg., 48, 1990, 617.

[2]. McHenry MC, Weinstein AJ, Antimicrobial drugs and infections in ambulatory patients: Some problems and perspectives, Med. Clin. North Am., 3, 1983, 67.

[3]. Peterson LJ, Booth DF, Efficacy of antibiotic prophylaxis in intraoral orthognathic surgery, J Oral Surg. $34,1976,1088$.

[4]. Paterson JA, Cardo VA, Stratigos GT, An examination of antibiotic prophylaxis in oral and maxillofacial surgery, J Oral Surg., $28,1970,753$

[5]. Alanis A, Weinstein AJ, Adverse reactions associated with the use of oral penicillins and cephalosporins, Med Clin North Am., 67, $1983 ; 113$.

[6]. Dajani AS, Bisno AL, Chung KJ, et al, Prevention of bacterial endocarditis: Recommendations of the American Heart Association, J Am Dent Assoc. 64, 1990; 2919. 
[7]. Dodson TB, Perrott DH, Gongloff R K, et al, Human immunodeficiency virus sero status and the risk of post-extraction complications, Int. J Oral Maxillofac. Surg., 23, 1994; 2, 100.

[8]. Council on Dental Therapeutics: Management of dental patients with prosthetic joints, J Am Dent Assoc., $121,1990,551$.

[9]. Simmons NA, Ball AP, Cawson RA, et al, Case against antibiotic prophylaxis for dental treatment of patients with joint prostheses, Lancet, 339, 1992, 301.

[10]. Shay K, Lloyd P, Dental schools: Practices of prophylactic antibiotic coverage for patients with prosthetic joints, J Dent Educ., 52, $1988,564$.

[11]. Jacobson JJ, Schweitzer S, De Porter DJ, et al, Chemoprophylaxis of dental patients with prosthetic joints: A simulation model, J Dent Educ., 52, 1988, 599 .

[12]. Cowley G, Too much of a good thing, Newsweek 13(50), 1994, 123.

[13]. Begley S, The end of antibiotics, Newsweek, 13(48), 1994, 123.

[14]. Kruger G, Impacted teeth, in Kruger G (ed): Textbook of Oral Surgery (ed 4), St Louis, MO, Mosby, 1974,84

[15]. Archer WH, Oral and Maxillofacial Surgery, Philadelphia, PA, Saunders, 1975, 414.

[16]. Marciani R, Antibiotics for head and neck infections, in Peterson LJ (ed): Principles of Oral and Maxillofacial Surgery, Philadelphia, PA, Lippincott, 1992, 206-207.

[17]. Alling CA, Impacted teeth, in Hardin J (ed): Clark's Clinical Dentistry, Philadelphia, PA, Lippincott, $1993,11$.

[18]. Curran JB, Kennett S, Young AR, An Assessment of the use of prophylactic antibiotics in third molar surgery, Int. J Oral Surg.,3, $1974,1$.

[19]. Happonen RP, Backstrom AC, Ylipaavalniemi P, Prophylactic use of Phenoxymethylpenicillin and tinidazole in mandibular third molar surgery, Br J Oral Maxillofac Surg., 28, 1990, 12.

[20]. Goldberg MH, Nemarich AN, Marco WP, Complications after mandibular third molar Surgery: A statistical analysis of 500 consecutive procedures in private practice, J Am Dent Assoc., 111, 1985, 277.

[21]. Capuzzi P, Montebugnoli L, Vaccaro MA, Extraction of impacted third molars: A Longitudinal prospective study, Oral Surg., 77, $1994,341$.

[22]. Burke JF, The effective period of preventive antibiotic action in experimental incisions and dermal lesions, Surgery 50, $1961,161$.

[23]. Laskin DM, Prophylactic antibiotics: A problem or panacea? , J Oral Surg., 34, 1976, 585.

[24]. Mac Gregor AJ, Anti-prophylactic antibiotics, J Oral Surg., 34, 1976, 1063.

[25]. Hall HD, Bildman BS, Hand CD, Prevention of dry socket with local application of tetracycline, J Oral Surg., $29,1971,35$.

[26]. Swanson AE, A double-blind study on the effectiveness of tetracycline in reducing the incidence of fibrinolytic alveolitis, J. Oral Maxillofac. Surg., 47, 1989, 165.

[27]. Rood JP, Murgatroyd J, Metronidazole in the prevention of dry socket, Br. J.Oral Surg. 17, $1979,62$.

[28]. Fridrich KL, Olson RAJ, Alveolar osteitis following surgical removal of mandibular third Molars, Anesth Progr., $37,1990,32$.

[29]. Nordenram A, Sydnes G, Odegard J, Neomycin-bacitracin cones in impacted third molar Sockets, Int. J Oral Surg., 2, 1973 , 279.

[30]. Mitchell DA, A controlled clinical trial of prophylactic tinidazole for Chemoprophylaxis in third molar surgery, Br Dent J., 160, 1986, 284

[31]. Mitchell DA, Morris TA, Tinidazole or pivampicillin in third molar surgery, Int. J Oral Maxillofac. Surg., $16,1987,171$.

[32]. Hellem S, Nordenram A, Prevention of postoperative symptoms by general antibiotic treatment and local bandage in removal of mandibular third molars, Int. J Oral Surg.2, 1973, 273.

[33]. MacGregor AJ, Addy A,Value of penicillin in the prevention of pain, swelling and trismus following the removal of ectopic mandibular third molars, lnt. J Oral Surg., 9, 1980, 166.

[34]. Bystedt H, Nord CE, Effect of antibiotic treatment on postoperative infection after surgical removal of mandibular third molars, Sweden Dent J., 4, 1980, 27.

[35]. Bystedt H, von Konow L, Nord CE, A comparison of the effect of phenoxymethylpenicillin and azidocillin on post-operative complications after surgical removal of impacted mandibular third molars, Sweden Dent J., 5 1981, 225.

[36]. Krekmanov L, Hollander HO, Relationship between bacterial contamination and alveolitis after third molar surgery, Int. J Oral Surgery, 9, 1980, 274. 